\title{
Minimax Location Problems With Nonlinear Costs*
}

\author{
P. M. Dearing** \\ (May 16, 1977)
}

\begin{abstract}
Previous studies of one-facility minimax location problems are extended by permitting the cost of travel to be given by any (strictly) increasing, continuous function of travel distance. Previous solution procedures for the rectilinear distance problem in the plane and for the problem on a tree network are extended to these general cost functions.
\end{abstract}

Key words: Facility location; location theory; minimax; networks.

\section{Introduction}

Minimax location problems have received considerable attention in the literature as models for locating facilities that are to provide emergency or convenient service to a set of existing facilities. In most of these problems there is given a set of existing facilities whose locations are represented as points in some space, and new facility locations are also to be specified as points in that space. A distance function is chosen to represent the travel distance between the new and existing facility locations. The minimax objective is to locate the new facilities so that the maximum distance, or a function of distance, between the new and existing facility locations is minimized.

Different problems may be specified by the choice of the space and of the distance function used. Most of the problems in the literature may be placed into one of two classes: those using a norm-derived distance in the space $R^{n}$ for some $n$, and those on a network using network distances. Also, different problems may be specified by the choice of the cost-representing functions of travel distance. These functions are referred to generically as "cost" functions, but they may measure cost, time or some other form of inconvenience.

This paper considers one-facility minimax location problems that permit quite general cost functions, namely any continuous (strictly) increasing function of the travel distance. In the previous considerations of minimax location problems, cost functions were assumed to be linear, or in many cases the identity function. The initial formulation and analysis are given for a problem in $R^{n}$ using norm-derived distances; however, the results obtained also hold for problems on a network and are discussed subsequently. In addition, for minimax location problems using rectilinear distance, this paper extends previous solution procedures to these more general cost functions in the space $R^{2}$. For the problem on a tree network, previous solution procedures are also extended to the general cost functions.

Most of the literature on minimax location problems has appeared in the last ten years, although one version of the problem was first formulated in 1857 as a "minimum covering sphere problem." A brief historical review is given by Elzinga and Hearn [6] ${ }^{1}$ who deal with the case involving the Euclidean distance in $R^{n}$ and the identity cost function. Elzinga and Hearn [5] and Nair and Chandrasekaran [23] develop additional solution procedures for the same problem in $R^{2}$. A multi-facility version of the Euclidean distance problem in $R^{2}$ is considered as a convex programming problem by Love, Wesolowsky and Kraemer [20].

Geometrical properties motivate solutions by Elzinga and Hearn [5] and by Francis [8] to a one-facility rectilinear distance problem in $R^{2}$ using the identity cost function. Wesolowsky [24], and Dearing and Francis [2] solve a multi-facility version of the rectilinear distance problem in $R^{2}$.

One-facility minimax location problems on a network with linear cost functions were first considered by Hakimi [12], [13] and subsequently by Goldman [11], Dearing and Francis [3] and Handler [16]. For tree networks the problem has been solved by Goldman [11], Handler [15], [16], Halfin [14], and Dearing and Francis [3]. A generalization for locating several new facilities, called the " $p$-center" problem, has been considered by Christofides and Viola [1], Minieka [22], Garfinkel et al. [10], and Handler [16]. Multi-facility minimax problems on tree networks are studied by Dearing, Francis, and Lowe [4].

\footnotetext{
$*$ An invited paper.
This research was partially accomplished while the author was a summer faculty employee in the Applied Mathematics Division, National Bureau of Standards, Washington, D. C.

** Present address: Department of Mathematical Sciences, Clemson University, Clemson, South Carolina 29631
} 
One-facility minimax location problems in $R^{n}$ using norm-derived distances and linear cost functions were studied by Francis [7]. The analysis and developments of that work motivate many of the extensions in this paper. A general reference on facility location is the book by Francis and White [9].

\section{Formulation and Analysis}

To state the minimax location problem under consideration, suppose that one new facility is to be located at a point $X=\left(x_{1}, \ldots, x_{n}\right)$ in $R^{n}$, and suppose that $m$ given points $P_{i}, 1 \leq i \leq m$, in $R^{n}$ represent the locations of existing facilities. Let $d(X, Y)$ denote a norm-derived distance between the points $X$ and $Y$ and suppose that $d(X, Y)$ is convex and continuous in $X$ for each fixed $Y$. For example, this includes distances of the form

$$
d(X, Y)=\left[\sum_{j=1}^{n}\left|x_{j}-y_{j}\right|^{p}\right]^{1 / p}
$$

for some real $p \geq 1$. When referring to a particular such distance for a specified $p$ we write $d_{p}(X, Y)$.

For $1 \leq i \leq m$, let $f_{i}$ be a continuous (strictly) increasing function with domain $[0, \infty)$. In particular apply $f_{i}$ to the distance $d\left(X, P_{i}\right)$ of $X$ from $P_{i}$ and define the function $f$ on $R^{n}$ by

$$
f(X)=\max _{1 \leq i \leq m}\left\{f_{i}\left[d\left(X, P_{i}\right)\right]\right\}
$$

The problem of interest, denoted by $P O$, is

$$
\min _{X \varepsilon R^{n}} f(X)
$$

Problem $P O$ may have a variety of applications depending on the dimension of the space $R^{n}$, the distance $d$ and the functions $f_{i}$. For example, suppose the existing facilities are locations of demand for emergency service and are represented as points in the plane $R^{2}$. Suppose that travel distance is approximated by the Euclidean distance $(p=2)$, and the functions $f_{i}$ are chosen to express the time of travel as a function of the distance from $P_{i}$. Then the function $f$ expresses the maximum time of travel between the new facility location $X$ and any existing facility.

In a recent study for the New York City Fire Department, Kolesar et al. [18] conclude from empirical observations that in most situations the time of travel for fire trucks is a particular continuous, nonlinear, increasing function of the travel distance. The assumptions made about the cost functions $f_{i}$ allow such representations of travel time as a function of travel distance. Also, freight movements often involve transport costs which are concave increasing functions of transport distance; these situations also may be represented by the $f_{i}$.

Next, some properties of the function $f$ will be observed and proved, including the existence of a point $X^{*}$ that minimizes $f$, the strict quasiconvexity of $f$, a lower bound for $f$, some conditions for the bound to be tight, and a necessary condition for a minimum solution. Most of these properties of $f$ are motivated by, and are extensions to, similar developments in [7].

Property 1: There exists an $\mathrm{X}^{*}$ in $\mathrm{R}^{\mathrm{n}}$ such that $\mathrm{f}\left(\mathrm{X}^{*}\right) \leq \mathrm{f}(\mathrm{X})$ for all $\mathrm{X}$ in $\mathrm{R}^{\mathrm{n}}$.

Proof: For each $i, 1 \leq i \leq m, f_{i}\left[d\left(X, P_{i}\right)\right]$ is a continuous function of $X$ since it is the composition of $f_{i}$ and the continuous function $d\left(P_{i}, X\right)$ of $X$. Thus $f$, the maximum of finitely many continuous functions, is continuous on $R^{n}$. It can be shown that the level set $S_{k}=\left\{X \in R^{n}: f(X) \leq k\right\}$, for any value of $k$ in the range of $f$, is compact in $R^{n}$. Thus there exists an $X^{*}$ in $S_{k}$ such that $f\left(X^{*}\right) \leq f(X)$ for all $X$ in $S_{k}$. By the definition of $S_{k}$, $f\left(X^{*}\right) \leq f(X)$ for all $X$ in $R^{n}$.

In the following property, the definition of strict quasiconvexity is taken from Mangasarian [21].

PROPERTY 2: The function $\mathrm{f}$ is strict quasiconvex on $\mathrm{R}^{\mathrm{n}}$. 
Proof: Let $X_{1}$ and $X_{2}$ be any two points in $R^{n}$ with $f\left(X_{1}\right)<f\left(X_{2}\right)$. We must show that $f\left(\lambda X_{1}+(1-\lambda) X_{2}\right)<$ $f\left(X_{2}\right)$ for $0<\lambda<1$. By the convexity of $d$ and the monotonicity of $f_{i}$, we have for some $s, f\left(\lambda X_{1}+(1-\lambda) X_{2}\right)$ $=f_{s}\left[d\left(\lambda X_{1}+(1-\lambda) X_{2}, P_{s}\right)\right] \leq f_{s}\left[\lambda d\left(X_{1}, P_{s}\right)+(1-\lambda) d\left(X_{2}, P_{s}\right)\right] \leq f_{s}\left[\max \left\{d\left(X_{1}, P_{s}\right), d\left(X_{2}, P_{s}\right)\right\}\right]=$ $\max \left\{f_{s}\left[d\left(X_{1}, P_{s}\right)\right], f_{s}\left[d\left(X_{2}, P_{s}\right)\right]\right\} \leq \max \left\{f\left(X_{1}\right), f\left(X_{2}\right)\right\}=f\left(X_{2}\right)$. If equality holds in the above, then $\lambda d\left(X_{1}, P_{s}\right)$ $+(1-\lambda) d\left(X_{2}, P_{s}\right)=\max \left\{d\left(X_{1}, P_{s}\right), d\left(X_{2}, P_{s}\right)\right\}$, and this implies $d\left(X_{1}, P_{s}\right)=d\left(X_{2}, P_{s}\right)$. Also equality implies $f\left(X_{2}\right)=f_{s}\left[d\left(X_{2}, P_{s}\right)\right]$. Then $f\left(X_{1}\right) \geq f_{s}\left[d\left(X_{1}, P_{s}\right)\right]=f_{s}\left[d\left(X_{2}, P_{s}\right)\right]=f\left(X_{2}\right)$, a contradiction, so that strict inequality holds.

Corollary: If $\mathrm{X}^{*}$ is a local minimum of $\mathrm{f}$, then $\mathrm{X}^{*}$ is a global minimum of $\mathrm{f}$.

Since each $f_{i}$ is continuous and increasing, $f_{i}^{-1}$ as well as $\left(f_{i}^{-1}+f_{j}^{-1}\right)$ and $\left(f_{i}^{-1}+f_{j}^{-1}\right)^{-1}$ is continuous and increasing. These observations are used next to develop a lower bound for the function $f$ and conditions for the bound to equal the value of $f$ at some point. Such a point is of course a solution to problem PO.

For each pair of existing facility locations $P_{i}$ and $P_{j}$, consider the function $\left(f_{i}^{-1}+f_{j}^{-1}\right)^{-1}$. We define $b(i$, $j)$ as that function's value at $d\left(P_{i}, P_{j}\right)$, provided $d\left(P_{i}, P_{j}\right)$ is in the domain, i.e.,

$$
b(i, j)=\left(f_{i}^{-1}+f_{j}^{-1}\right)^{-1}\left[d\left(P_{i}, P_{j}\right)\right] .
$$

Only the values $b(i, j)$ are used in obtaining the lower bound. We next describe the domain of $\left(f_{i}^{-1}+f_{j}^{-1}\right)^{-1}$ and give conditions for $b(i, j)$ to exist.

The domain of $\left(f_{i}^{-1}+f_{j}^{-1}\right)^{-1}$ is an interval whose end points are obtained as follows. The range of $f_{i}$ for each $i, 1 \leq i \leq m$, is the interval $\left[f_{i}(0), M_{i}\right)$ for some value of $M_{i}$ (finite or infinite), and so this interval is the domain of $f_{i}^{-1}$. The domain of $\left(f_{i}^{-1}+f_{j}^{-1}\right)$ is the intersection of $\left[f_{i}(0), M_{i}\right)$ and $\left[f_{j}(0), M_{j}\right)$, i.e., $\left[\max \left\{f_{i}(0)\right.\right.$, $\left.\left.f_{j}(0)\right\}, \min \left\{M_{i}, M_{j}\right\}\right)$ which we denote by $\left[m_{i j}, M_{i j}\right)$. This interval may be empty, in which case $\left(f_{i}^{-1}+f_{j}^{-1}\right)^{-1}$ is not defined. If this occurs because $M_{j} \leq f_{i}(0)$, then for all $X, f_{j}\left[d\left(X, P_{j}\right)\right]<M_{j} \leq f_{i}(0) \leq f_{i}\left[d\left(X, P_{i}\right)\right]$. In this case the point $P_{j}$ and the function $f_{j}$ may be deleted from the problem since they do not enter into the definition of $f(X)$. Similarly $P_{i}$ and $f_{i}$ may be deleted if $M_{i} \leq f_{j}(0)$. We therefore assume, for the remainder of the paper, that the intervals $\left[m_{i j}, M_{i j}\right)$ are nonempty for all $1 \leq i \neq j \leq m$.

The range of $\left(f_{i}^{-1}+f_{j}^{-1}\right)$ is the domain of $\left(f_{i}^{-1}+f_{j}^{-1}\right)^{-1}$, i.e., $\left[\left(f_{i}^{-1}+f_{j}^{-1}\right)\left[m_{i j}\right],\left(f_{i}^{-1}+f_{j}^{-1}\right)\left[M_{i j}\right]\right)$. Using the definition of $m_{i j}$ and observing that $f_{i}^{-1}\left[f_{i}(0)\right]\left[f_{j}^{-1}\left[f_{j}(0)\right]=0\right.$, the left end point of this interval can be written as $\max \left\{f_{j}^{-1}\left[f_{i}(0)\right], f_{i}^{-1}\left[f_{j}(0)\right]\right\}$. Similarly, using the definition of $M_{i j}$ and observing that $f_{i}^{-1}\left(M_{i}\right)=$ $f_{j}^{-1}\left(M_{j}\right)=\infty$, the right end point of this interval is seen to be $\infty$. Thus the domain of $\left(f_{i}^{-1}+f_{j}^{-1}\right)^{-1}$ is the interval $\left[\max \left\{f_{i}^{-1}\left[f_{j}(0)\right], f_{j}^{-1}\left[f_{i}(0)\right]\right\}, \infty\right)$. (For a bounded space, with diameter say $M$, we replace $\infty$ by $M$ in the above development.) This yields the following remark which gives a way to check whether $b(i, j)$ is defined.

Remark 1: $\mathrm{d}\left(\mathrm{P}_{\mathrm{i}}, \mathrm{P}_{\mathrm{j}}\right)$ is in the domain of $\left(\mathrm{f}_{\mathrm{i}}^{-1}+\mathrm{f}_{\mathrm{j}}^{-1}\right)^{-1}$ if and only if $\mathrm{f}_{\mathrm{j}}\left[\mathrm{d}\left(\mathrm{P}_{\mathrm{i}}, \mathrm{P}_{\mathrm{j}}\right)\right] \geq \mathrm{f}_{\mathrm{i}}(0)$ and $\mathrm{f}_{\mathrm{i}}\left[\mathrm{d}\left(\mathrm{P}_{\mathrm{i}}, \mathrm{P}_{\mathrm{j}}\right)\right]$ $\geq \mathrm{f}_{\mathrm{j}}(0)$.

Next let $D$ be the set of unordered index pairs $(i, j)$ such that $b(i, j)$ is defined. Define

$$
B=\max _{(i, j) \in D} b(i, j) .
$$

We will show that $B$ is a lower bound for $f$. We often write $B=b(s, t)$ (or $b(s, s)$ ) to specify that the indices $s$ and $t$ (or $s$ ) give the maximum value of $b(i, j)$. Also, for $i=j, b(i, i)=f_{i}(0)$, and so an equivalent expression for $B$ is

$$
B=\max \left\{\max _{\substack{(i, j) \in D \\ i \neq j}} b(i, j), \max _{1 \leq i \leq m} f_{i}(0)\right\} .
$$

Clearly the function $\left(f_{\boldsymbol{i}}^{-1}+f_{j}^{-1}\right)^{-1}$ may be difficult to determine for general functions $f_{i}$ and $f_{j}$. However, if the $f_{i}$ are piecewise linear approximations to the actual cost functions, the expressions $b(i, j)$ may be determined explicitly. As an example, suppose $\operatorname{each} f_{i}$ is concave piecewise linear function with domain $[0, \infty)$ and is specified by the $k_{i}$ linear expressions $\alpha_{s}^{i} x+\beta_{s}^{i}$ for $1 \leq s \leq k_{i}$ with 


$$
f_{i}(x)=\min _{1 \leq s \leq k_{i}}\left\{\alpha_{s}^{i} x+\beta_{s}^{i}\right\}
$$

Then $f_{i}^{-1}(y)=\max _{1 \leq s \leq k_{i}}\left\{\left(y-\beta_{s}^{i}\right) / \alpha_{s}^{i}\right\}$, and for each pair $(i, j)$ in $D$

$$
\left(f_{i}^{-1}+f_{j}^{-1}\right)(y)=\max _{\substack{1 \leq s \leq k_{i} \\ 1 \leq t \leq k_{j}}}\left\{\left[\left(\alpha_{s}^{i}+\alpha_{t}^{j}\right) y-\left(\beta_{s}^{i} \alpha_{t}^{j}+\beta_{t}^{j} \alpha_{s}^{i}\right)\right] / \alpha_{s}^{i} \alpha_{t}^{\}}\right\} .
$$

Finally,

$$
\left(f_{i}^{-1}+f_{j}^{-1}\right)^{-1}(x)=\min _{\substack{1 \leq s \leq k_{i} \\ 1 \leq t \leq k_{j}}}\left\{\left(\alpha_{s}^{i} \alpha_{t}^{j} x+\beta_{s}^{i} \alpha_{t}^{j}+\beta_{t}^{j} \alpha_{s}^{i}\right) /\left(\alpha_{s}^{i}+\alpha_{t}^{j}\right)\right\}
$$

and $b(i, j)$ is obtained from the last expression with $x=d\left(P_{i}, P_{j}\right)$.

Some further relationships between $B$ and $f$ are now given.

Property 3: For all $\mathrm{X}$ in $\mathrm{R}^{\mathrm{n}}, \mathrm{B} \leq \mathrm{f}(\mathrm{X})$.

Proof: For $1 \leq i \leq m, b(i, i)=f_{i}\left[d\left(P_{i}, P_{i}\right)\right] \leq f_{i}\left[d\left(P_{i}, X\right)\right] \leq f(X)$. Next consider $b(i, j)$ for any $i \neq j$ in $D$. Let $X^{\prime}$ minimize max $\left\{f_{i}\left[d\left(P_{i}, X\right)\right], f_{j}\left[d\left(P_{j}, X\right)\right]\right\}$ and suppose $f_{j}\left[d\left(P_{j}, X^{\prime}\right)\right] \geq f_{i}\left[d\left(P_{i}, X^{\prime}\right)\right]$. ( $X^{\prime}$ exists by Property 1.) It can be shown that $f_{j}\left[d\left(P_{j}, X^{\prime}\right)\right]$ is in the domain of $f_{i}^{-1}$, using the existence of $b(i, j)$. Then

$$
\begin{aligned}
b(i, j) & =\left(f_{i}^{-1}+f_{j}^{-1}\right)^{-1}\left[d\left(P_{i}, P_{j}\right)\right] \leq\left(f_{i}^{-1}+f_{j}^{-1}\right)^{-1}\left[d\left(P_{i}, X^{\prime}\right)+d\left(P_{j}, X\right)\right] \\
& =\left(f_{i}^{-1}+f_{j}^{-1}\right)^{-1} \cdot\left(f_{i}^{-1} f_{i}\left[d\left(P_{i}, X^{\prime}\right)\right]+f_{j}^{-1} f_{j}\left[d\left(P_{j}, X^{\prime}\right)\right]\right) \\
& \leq\left(f_{i}^{-1}+f_{j}^{-1}\right)^{-1} \cdot\left(f_{i}^{-1} f_{j}\left[d\left(P_{j}, X^{\prime}\right)\right]+f_{j}^{-1} f_{j}\left[d\left(P_{j}, X^{\prime}\right)\right]\right) \\
& =\left(f_{i}^{-1}+f_{j}^{-1}\right)^{-1} \cdot\left(f_{i}^{-1}+f_{j}^{-1}\right) \cdot f_{j}\left[d\left(P_{j}, X^{\prime}\right)\right]=f_{j}\left[d\left(P_{j}, X^{\prime}\right)\right] \\
& \leq \max \left\{f_{i}\left[d\left(P_{i}, X\right)\right], f_{j}\left[d\left(P_{j}, X\right)\right]\right\} \quad \text { for all } X .
\end{aligned}
$$

If $f_{j}\left[d\left(P_{j}, X^{\prime}\right)\right] \leq f_{i}\left[d\left(P_{i}, X^{\prime}\right)\right]$, we get the same result.

Finally,

$$
B=\max _{(i, j) \in D} b(i, j) \leq \max _{(i, j) \in D} \max \left\{f_{i}\left[d\left(P_{i}, X\right)\right], f_{j}\left[d\left(P_{j}, X\right)\right]\right\} \leq f(X)
$$

The next two properties give conditions for $B$ to equal $f(X)$ for some point $X$ in $R^{n}$.

Property 4: $\quad \mathrm{B}=\mathrm{b}(\mathrm{s}, \mathrm{t})=\mathrm{f}(\mathrm{X})$ if and only if the points $\mathrm{P}_{\mathrm{s}}, \mathrm{P}_{\mathrm{t}}$, and $\mathrm{X}$ satisfy

(a) $d\left(P_{s}, P_{t}\right)=d\left(P_{s}, X\right)+d\left(X, P_{t}\right)$

(b) $f_{s}\left[d\left(P_{s}, X\right)\right]=f_{t}\left[d\left(P_{t}, X\right)\right]$

(c) $B \geq f_{i}\left[d\left(P_{i}, X\right)\right] \quad$ for all $\quad i \neq s, t$.

The proof of Property 4 follows from the proof of Property 3 . In particular, (a) and (b) imply $f_{s}\left[d\left(P_{s}, P_{t}\right)\right] \geq$ $f_{s}\left[d\left(P_{s}, X\right)\right]=f_{t}\left[d\left(P_{t}, X\right)\right] \geq f_{t}(0)$ and similarly $f_{t}\left[d\left(P_{s}, P_{t}\right)\right] \geq f_{s}(0)$, so that Remark 1 applies to assure that $b(s, t)$ is defined.

Property 5: $\mathrm{B}=\mathrm{f}_{\mathrm{s}}\left[\mathrm{d}\left(\mathrm{P}_{\mathrm{s}}, \mathrm{P}_{\mathrm{s}}\right)\right]=\mathrm{f}_{\mathrm{s}}(0)$ for some point $\mathrm{P}_{\mathrm{s}}$ if and only if $\mathrm{f}\left(\mathrm{P}_{\mathrm{s}}\right)=\mathrm{f}_{\mathrm{s}}\left[\mathrm{d}\left(\mathrm{P}_{\mathrm{s}}, \mathrm{P}_{\mathrm{s}}\right)\right]$; in either case $\mathrm{P}_{\mathrm{s}}$ is the unique minimum point of $\mathrm{f}$. 
Proof: Suppose $f\left(P_{s}\right)=f_{s}\left[d\left(P_{s}, P_{s}\right)\right]$. Then

$$
B \leq f\left(P_{s}\right)=f_{s}(0) \leq B \text { implies } B=f_{s}\left[d\left(P_{s}, P_{s}\right)\right]=f\left(P_{s}\right) .
$$

Suppose $B=f_{s}\left[d\left(P_{s}, P_{s}\right)\right]=f_{s}(0)$ but that for some $i, f_{s}\left[d\left(P_{s}, P_{s}\right)\right]<f_{i}\left[d\left(P_{s}, P_{i}\right)\right]=f\left(P_{s}\right)$. Since $B=$ $f_{s}(0), f_{s}(0) \geq f_{i}(0)$ so that $f_{i}(0) \leq f_{s}(0) \leq f_{s}\left[d\left(P_{s}, P_{i}\right)\right]$. Thus Remark 1 implies that $b(s, i)$ exists. By assumption $B=f_{s}(0) \geq b(s, i)=\left(f_{s}^{-1}+f_{i}^{-1}\right)^{-1}\left[d\left(P_{s}, P_{i}\right)\right]$. Also, $f_{i}(0) \leq f_{s}(0)<f_{i}\left[d\left(P_{s}, P_{i}\right)\right]<M_{i}$ implies that $f_{s}(0)$ is in the domain of $\left(f_{s}^{-1}+f_{i}^{-1}\right)$, and we have $\left(f_{s}^{-1}+f_{i}^{-1}\right)\left[f_{s}(0)\right] \geq d\left(P_{s}, P_{i}\right)$. This implies $f_{i}^{-1}\left[f_{s}(0)\right] \geq d\left(P_{s}, P_{i}\right)$, or $f_{s}(0) \geq f_{i}\left[d\left(P_{s}, P_{i}\right)\right]$ which is a contradiction. Thus $f_{s}(0) \geq f_{i}\left[d\left(P_{s}, P_{i}\right)\right]$ for all $i$ and $f\left(P_{s}\right)=f_{s}(0)=B$.

In either case $f\left(P_{s}\right)=B$ so that $P_{s}$ minimizes $f$. To prove uniqueness, suppose $\mathrm{X}^{\circ}$ minimizes $f$. Then $B=$ $f_{s}\left[d\left(P_{s}, P_{s}\right)\right] \leq f_{s}\left[d\left(P_{s}, X^{\circ}\right)\right] \leq f\left(X^{\circ}\right)=B$ which implies $d\left(P_{s}, X^{\circ}\right)=0$ and $X^{\circ}=P_{s}$.

The next property considers the case where $B>f_{i}(0)$ for $1 \leq i \leq m$, and provides a necessary condition for a point $X^{*}$ to minimize $f$. The proof of this property is analogous to the proof of a similar result in reference [3], and is not given here.

Property 6: Let $\mathrm{X}^{*}$ minimize $\mathrm{f}$ and suppose that $\mathrm{B}>\mathrm{f}_{\mathrm{i}}(0), \mathrm{l} \leq \mathrm{i} \leq \mathrm{m}$. Then there exist distinct points $\mathrm{P}_{\mathrm{s}}$ and $\mathrm{P}_{\mathrm{t}}$ such that $\mathrm{f}\left(\mathrm{X}^{*}\right)=\mathrm{f}_{\mathrm{s}}\left[\mathrm{d}\left(\mathrm{P}_{\mathrm{s}}, \mathrm{X}^{*}\right)\right]=\mathrm{f}_{\mathrm{t}}\left[\mathrm{d}\left(\mathrm{P}_{\mathrm{t}}, \mathrm{X}^{*}\right)\right]$.

Since $B$ is a lower bound for $f$, if there is a point $X$ in $R^{n}$ such that $f(X) \leq B$, then $X$ minimizes $f$. For two special cases we show that the set $S_{B}=\left\{X \in R^{n}: f(X) \leq B\right\}$ is not empty, and we characterize all the points in $S_{B}$. In characterizing $S_{B}$ it is useful to write $S_{B}=\bigcap_{1 \leq i \leq m} A_{i}$ where $A_{i}=\left\{X \in R^{n}: f_{i}\left[d\left(X, P_{i}\right)\right] \leq B\right\}, 1 \leq i \leq m$. The following lemma is used later but is stated here since it holds for any norm-derived distance in $R^{n}$.

Lemma 1: For each pair of indices $\mathrm{i}, \mathrm{j}$ with $\mathrm{l} \leq \mathrm{i}, \mathrm{j} \leq \mathrm{m}, \mathrm{A}_{\mathrm{i}} \cap \mathrm{A}_{\mathrm{j}} \neq \phi$.

Proof: Observe that $P_{i} \in A_{i}$ for each $1 \leq i \leq m$, since $f_{i}\left[d\left(P_{i}, P_{i}\right)\right] \leq B$. Thus for $i=j$, the conclusion holds. For $i \neq j$ there are three cases.

1. $f_{i}(0)>f_{j}\left[d\left(P_{i}, P_{j}\right)\right]$ : This implies $P_{i} \epsilon A_{j}$ since $f_{i}(0) \leq B$.

2. $f_{i}(0)>f_{i}\left[d\left(P_{\mathrm{i}}, P_{\mathrm{j}}\right)\right]$ : Thus $P_{j} \in A_{i}$.

3. $f_{i}(0) \leq f_{j}\left[d\left(P_{i}, P_{j}\right)\right]$ and $f_{j}(0) \leq f_{i}\left[d\left(P_{i}, P_{j}\right)\right]$ : This implies $b(i, j)=$

$$
\left(f_{i}^{-1}+f_{\mathrm{j}}^{-1}\right)^{-1}\left[d\left(P_{\mathrm{i}}, P_{\mathrm{j}}\right)\right] \text { or that } f_{i}^{-1}[b(i, j)]+f_{j}^{-1}[b(i, j)]=d\left(P_{i}, P_{j}\right) .
$$

Each summand on the left-hand side is nonnegative so $f_{i}^{-1}[b(i, j)] \leq d\left(P_{i}, P_{j}\right)$. By the intermediate value theorem, there exists a point $X_{0}$ on the line segment between $P_{i}$ and $P_{j}$ such that $d\left(X_{0}, P_{i}\right)=f_{i}^{-1}[b(i, j)]$. Substituting this expression together with $d\left(P_{i}, P_{j}\right)=d\left(P_{i}, X_{0}\right)+d\left(X_{0}, P_{j}\right)$ into the last equation gives $d\left(X_{0}\right.$, $\left.P_{j}\right)=f_{j}^{-1}[b(i, j)]$. Thus $f_{i}\left[d\left(X_{0}, P_{i}\right)\right]=f_{j}\left[d\left[\left(X_{0}, P_{j}\right)\right]=b(i, j)\right.$ and since $b(i, j) \leq B, X_{0}$ is in $A_{i}$ and $A_{j}$. This proves the lemma.

\section{The Rectilinear Distance Problem in $\mathbf{R}^{2}$}

A special case of problem $P 0$ is considered that uses the rectilinear distance in $R^{2}$. The rectilinear distance is denoted by $d_{1}\left(X, P_{i}\right)$ and is defined by taking $p=1$ in $(1)$. For the case where the cost functions are linear functions of distance, an explicit solution procedure is reported in reference [9]. This procedure is extended here to the functions $f_{i}$ of distance.

Note that if $B$ is not in the range of $f_{i}$, then $f_{i}\left[d_{1}\left(X, P_{i}\right)\right] \leq B$ for all $X \in R^{2}$ and $A_{i}=R^{2}$. Otherwise $B$ is in the domain of $f_{i}^{-1}$ and $A_{i}=\left\{X \in R^{2}: d_{1}\left(X, \mathrm{P}_{i}\right) \leq f_{i}^{-1}(B)\right\}$. Let $E$ be the index set of all $i$ such that $B$ is in the domain of $f_{i}^{-1}$. Thus $S_{B}=\bigcap A_{i}$.

Using the rectilinear distance in $R^{2}$, each set $A_{i}, i \in E$ may be further characterized, as in reference [9], by a system of linear inequalities. Denoting $X=(x, y)$ and $P_{i}=\left(a_{i}, b_{i}\right)$, we may write 


$$
\begin{array}{r}
A_{i}=\left\{(x, y) \in R^{2}:-f_{i}^{-1}(B)+a_{i}+b_{i} \leq x+y \leq f_{i}^{-1}(B)+a_{i}+b_{i},\right. \\
\left.-f_{i}^{-1}(B)+a_{i}-b_{i} \leq x-y \leq f_{i}^{-1}(B)+a_{i}-b_{i}\right\} .
\end{array}
$$

Then $S_{B}=\bigcap_{i \in E} A_{i}$ implies that $S_{B}$ is the set of all points $(x, y)$ in $R^{2}$ such that

$$
\max _{i \in E}\left\{-f_{i}^{-1}(B)+a_{i}+b_{i}\right\} \leq x+y \leq \min _{i \in E}\left\{f_{i}^{-1}(B)+a_{i}+b_{i}\right\}
$$

and

$$
\max _{i \in E}\left\{-f_{i}^{-1}(B)+a_{i}-b_{i}\right\} \leq x-y \leq \min _{i \in E}\left\{f_{i}^{-1}(B)+a_{i}-b_{i}\right\} .
$$

The above inequalities imply that $S_{B}$ is nonempty if and only if

$$
-f_{i}^{-1}(B)+a_{i}+b_{i} \leq f_{j}^{-1}(B)+a_{j}+b_{j},
$$

and

$$
-f_{i}^{-1}(B)+a_{i}-b_{i} \leq f_{j}^{-1}(B)+a_{j}-b_{j}
$$

for all pairs $(i, j) i, j \in E$ or equivalently if

$$
d_{1}\left(P_{i}, P_{j}\right)=\left|a_{i}-a_{j}\right|+\left|b_{i}-b_{j}\right| \leq f_{i}^{-1}(B)+f_{j}^{-1}(B) .
$$

If $b(i, j)$ is defined, the above inequality holds since $b(i, j) \leq B$. If $b(i, j)$ is not defined, then either $B \geq$ $\mathrm{f}_{i}(0)>f_{j}\left[d_{1}\left(P_{i}, P_{j}\right)\right]$ or $B \geq f_{j}(0)>f_{i}\left[d_{1}\left(P_{i}, P_{j}\right)\right]$. The first case implies $f_{i}^{-1}(B) \geq 0$ and $f_{j}^{-1}(B)>d_{1}\left(P_{i}, P_{j}\right)$, and adding gives the desired inequality. The second case is similar. This proves the following result.

Property 7: Using the rectilinear distance in $\mathrm{R}^{2}, \mathrm{~B}=\min _{\mathrm{X} \in \mathrm{R}^{2}} \mathrm{f}(\mathrm{X})$.

Interestingly, the above solution procedure does not extend to the rectilinear problem in $R^{n}$ for $n>2$, that is, it is possible that $B<f(X)$ for all $X$ in $R^{n}$. As an example, consider existing facilities at the points $P_{1}=(1$, $0,0), P_{2}=(0,1,0), P_{3}=(0,0,1), P_{4}=(1,1,1)$ in $R^{3}$, and let $f_{i}$ be the identity function for $1 \leq i \leq 4$. Then $b(i, j)=1$ for all pairs of distinct $i$ and $j$ and all $b(i, i)=0$, so $B=1$. The sets $A_{i}$ in $R^{3}$ intersect pair-wise (in fact any three of the sets $A_{i}$ intersect), but the intersection of all four sets $A_{i}$ is empty.

To describe further why the bound $B$ may not be tight in $R^{n}$, we use the following set intersection property: a finite collection of sets is said to satisfy the pair-wise intersection property if the nonempty intersection of each pair of sets in the collection implies that the intersection of all sets in the collection is not empty. Observe that when using the rectilinear distance, each set $A_{i}$ is an octahedron in $R^{n}$ (with appropriate definition for $n>3$ ), and Lemma 1 implies that the $A_{i}$ intersect pair-wise. However, it is shown in reference [19] that octahedra in $R^{n}$ satisfy the pair-wise intersection property if and only if $n \leq 2$. The "if" part provides an alternate proof of Property 7.

\section{Minimax Problems on a Network}

Consider a minimax location problem with respect to a system of existing facility locations and given transportation links between the facilities. As in reference [3], such a system may be modeled as a network metric space $N$. The $m$ existing facility locations correspond to nodes $a_{i}, 1 \leq i \leq m$, of $N$, and transportation links correspond to edges of $N$. Each point along a given transportation link is a feasible location for a new facility and is represented by a point $x$ on the corresponding edge of $N$. For any two points $x$ and $y$ in $N$, the distance between $x$ and $y$, denoted by $d(x, y)$, is the length of a shortest path between $x$ and $y$, denoted by $P(x, y)$. For each $i, d\left(x, a_{i}\right)$ is a continuous function of $x$ on $N$ [3]. 
As above, let $f_{i}$ be an increasing, continuous function of distance and define $f_{N}$ on $N$ by

$$
f_{N}(x)=\max _{1 \leq i \leq m}\left\{f_{i}\left[d\left(x, a_{i}\right)\right]\right\},
$$

and the problem $P N$ by

$$
\min _{x \in N} \mathrm{f}_{N}(\mathrm{x})
$$

It is shown in reference [3] that $N$ is a compact metric space, and as in the proof of Property $1, f_{N}$ is continuous on $N$. Thus there exists a point $x^{*}$ in $N$ that minimizes $f_{N}$.

Next observe that a lower bound $B$ may be defined analogous to (2) and (3). It follows that $B$ is a lower bound for $f_{N}$ since the proof of Property 3 used only the triangle inequality. Properties 4,5 , and 6 also may be proved for $f_{N}$ by following the respective proofs above and using similar results in reference [3] for linear functions of distance.

For the special case of a tree network $T$, additional properties may be proved and a solution procedure obtained. Let $f_{T}$ denote the function defined on $T$ by expression (4). Observe that the proof of strict quasiconvexity of $f$ in Property 2 required only the convexity of $d$ and the assumption of increasing $f_{i}$. In reference [4], it is shown that the distance $d$ on a network is convex if and only if the network is a tree. Thus an argument similar to the proof of Property 2 shows that $f_{T}$ is strict quasiconvex.

Analogous to the above development, we may define the sets $A_{i}, 1 \leq i \leq m$ and the set $S_{B}=\underset{1 \leq i \leq m}{\bigcap} A_{i}$ for the problem on a tree. We may also show, analogous to Lemma 1, that each pair of sets $A_{i}$ and $A_{j}$ have nonempty intersection. It is shown in reference [17] that the pair-wise intersection property holds for connected subsets of a tree. This development implies that $S_{B}$ is not empty and we conclude that Property 7 holds for $f_{T}$, that is, $B=\min _{x \in T} f_{T}(x)$. This development also gives a procedure for minimizing $f_{T}$ : determine $B=$ $b(s, t)$, then the point $x^{*}$ such that $d\left(a_{s}, x^{*}\right)=f_{s}^{-1}(B)$ on the path $P\left(a_{s}, a_{t}\right)$ minimizes $f_{T}$. It may also be shown using results in reference [3] that $x^{*}$ is the unique minimum of $f_{T}$ on $T$.

In conclusion we note that for a general network $N$, the points that minimize $f_{N}$ may be characterized in terms of spanning trees of $N$. Let $T_{j}, 1 \leq j \leq \nu$, be all the spanning trees of $N$.

Property 8: A point $\mathrm{x} *$ in $\mathrm{N}$ minimizes $\mathrm{f}_{\mathrm{N}}$ if and only if $\mathrm{x} *$ minimizes $f_{\mathrm{T}_{\mathrm{k}}}$ for some $\mathrm{T}_{\mathrm{k}}$ such that $\min _{1 \leq j \leq \nu} \mathrm{B}\left(\mathrm{f}_{\mathrm{T}_{\mathrm{j}}}\right)=$ $\mathrm{B}\left(\mathrm{f}_{\mathrm{T}_{\mathrm{k}}}\right)$.

Using the above developments for $f_{N}$, the proof of Property 8 is a direct extension to the proof of a similar result in reference [3]. This property provides an interesting characterization of the minimum solutions of problem $P N$ in terms of the network structure. However, at the present no efficient solution procedures are known for solving PN, even for identity cost functions.

The author is grateful to A. J. Goldman for his helpful comments and suggestions during the course of this work and to the referees for their constructive comments, especially concerning the conditions for $b(i, j)$ to exist.

\section{References}

[1] Christofides, N., and Viola, P., The optimum location of multi-centres on a graph, Opnal. Res. Quart. 22, 145-154 (1971).

[2] Dearing, P. M., and Francis, R. L., A network flow solution to a multifacility minimax location problem involving rectilinear distances, Trans. Sci. 8, 126-141 (1974).

[3] Dearing, P. M., and Francis, R. L. A minimax location problem on a network, Trans. Sci. 8, 333-343, (1974).

[4] Dearing, P. M., Francis, R. L., and Lowe, T. J., Convex location problems on tree networks, Opns. Res. 24, 628-642, (1976).

[5] Elzinga, J., and Hearn, D., Geometrical solutions for some minimax location problems, Trans. Sci. 6, 379-394 (1972).

[6] Elzinga, J., and Hearn, D., The minimum covering sphere problem, Mng. Sci. 19, 96-104 (1972).

[7] Francis, R. L., Some aspects of a minimax location problem, Opns. Res. 15, 1163-1169 (1967).

[8] Francis, R. L., A geometrical solution procedure for a rectilinear distance minimax location problem, AIIE Trans. 4, 328-332 (1972).

[9] Francis, R. L., and White, J. A., Facility layout and location (Prentice-Hall, Englewood Cliffs, N.J., 1974). 
[10] Garfinkel, R. S., Neebe, A. W., and Rao, M. R., The $m$-center problem: bottleneck facility location, Working paper No. 7414, Graduate School of Management, University of Rochester, Rochester, N.Y. (1974).

[11] Goldman, A. J., Minimax location of a facility in a network, Trans. Sci. 6, 407-418 (1972).

[12] Hakimi, S. L., Optimum locations of switching centers and the absolute centers and medians of a graph, Opns. Res. 12, 450-459 (1964).

[13] Hakimi, S. L., Optimum distribution of switching centers in a communication network and some related graph theoretic problems, Opns. Res. 13, 462-475 (1965).

[14] Halfin, S., On finding the absolute and vertex centers of a tree with distances, Trans. Sci. 8, 75-77 (1974).

[15] Handler, G. Y., Minimax location of a facility in an undirected tree graph, Trans. Sci. 7, 287-293 (1973).

[16] Handler, G. Y., Minimax network location: theory and algorithms, Report FTL-R74-4, Flight Transportation Laboratory, M.I.T., Cambridge, Mass. (1974).

[17] Horn, W. A., Three results for trees, using mathematical induction, J. Res. Nat. Bur. stand. (U.S.), 76B (Math. Sci.), Nos. 1 \& 2 , 39-43 (Jan.-June 1972).

[18] Kolesar, P., Walker, W., and Hausner, J., Determining the relation between fire engine travel times and travel distances in New York City, Opns. Res. 23, 614-627, (1975).

[19] Lindenstrauss, J., Extensions of Compact Operations, Memoirs of the Am. Math. Soc. No. 48, (1964).

[20] Love, R. F., Wesolowsky, G. O., and Kraemer, S. A., A multifacility minimax location method for Euclidean distances, Int. J. of Prod. Res. 11, 37-45, (1973).

[21] Mangasarian, O. L., Nonlinear Programming, (McGraw-Hill, New York, 1969).

[22] Minieka, E., The $m$-center problem, SIAM Review, 12, 138-139 (1970).

[23] Nair, K. P. K., and Chandrasekaran, R., Optimal location of a single service center of certain types, Naval Res. Log. Quart. 18, 503-510 (1971).

[24] Wesolowsky, G. O., Rectangular distance location under the minimax optimality criterion, Trans. Sci. 6, $103-113$ (1972). 